Portland State University

PDXScholar

2007

\title{
Observation of Microslip Dynamics at High-Speed Microcontacts
}

\author{
Brian Borovsky \\ St. Olaf College \\ Adam M. Booth \\ Portland State University, boothad@pdx.edu \\ Erin Manlove \\ St. Olaf College
}

Follow this and additional works at: https://pdxscholar.library.pdx.edu/geology_fac

Part of the Geology Commons, and the Geomorphology Commons

Let us know how access to this document benefits you.

\section{Citation Details}

Borovsky, B., Booth, A., \& Manlove, E. (2007). Observation of microslip dynamics at high-speed microcontacts. Applied Physics Letters, 91(11), 114101.

This Article is brought to you for free and open access. It has been accepted for inclusion in Geology Faculty Publications and Presentations by an authorized administrator of PDXScholar. Please contact us if we can make this document more accessible: pdxscholar@pdx.edu. 


\title{
Observation of microslip dynamics at high-speed microcontacts
}

\author{
Brian Borovsky ${ }^{\text {a) }}$ \\ Department of Physics, St. Olaf College, Northfield, Minnesota 55057 \\ Adam Booth \\ Department of Physics, Grinnell College, Grinnell, Iowa 50112 \\ Erin Manlove \\ Department of Physics, St. Olaf College, Northfield, Minnesota 55057
}

(Received 28 June 2007; accepted 21 August 2007; published online 12 September 2007)

\begin{abstract}
The high-speed tribological properties of microscale contacts are studied using an indenter probe and quartz crystal microbalance. Elastic and dissipative shear forces are monitored as a function of contact radius for sapphire/gold interfaces with an adsorbed octadecanethiol monolayer. We observe shear force transitions understood as taking the interface from slipping to stuck conditions. We relate this behavior to the presence of interfacial microslip. Dynamic modeling shows that our observations are consistent with an interface that, when sliding, experiences full slip during half of each cycle. We discuss the implications for this technique as a sensitive probe of sliding friction.

(C) 2007 American Institute of Physics. [DOI: 10.1063/1.2784172]
\end{abstract}

The progressive miniaturization of mechanical devices sustains current interest in the field of micro/nanotribology. ${ }^{1}$ While a fundamental understanding of friction in dynamic contacts remains elusive, applications such as magnetic disk drives and microelectromechanical systems stand to benefit significantly from advances in this area. ${ }^{2}$ Sliding speeds in these devices routinely reach 1 to $10 \mathrm{~m} / \mathrm{s}$. This regime cannot be accessed by the most common nanotribological techniques, including the surface forces apparatus (SFA) and the atomic force microscope. ${ }^{3}$ These methods are limited to speeds less than $1 \mathrm{~mm} / \mathrm{s}$, and typically run far slower than this. One approach to bridging the speed gap has been to integrate an indenter probe, SFA, or other technique with a quartz crystal microbalance (QCM). ${ }^{4-8}$ The QCM provides surface velocities near $1 \mathrm{~m} / \mathrm{s}$ and sensitively detects elastic and dissipative interaction forces. This information is monitored through changes in the QCM resonant frequency $f$ and bandwidth $\Gamma$. Several challenges remain for approaches to the high-speed regime based on quartz oscillators. It is difficult to determine the state of relative motion at the reciprocating interface and to distinguish the mechanisms by which energy is dissipated. Recently, Berg and Johannsmann have observed peaks in the dissipation signal upon formation and disruption of contacts to a QCM surface. ${ }^{6}$ With an elegant series of measurements based on amplitude variation, these authors conclude that microslip dominates the interaction; the interface is partially stuck and partially slipping. This study was limited, however, to the initial stages of contact formation, and it was not possible to quantify the normal load or contact radius.

In this letter, we report on the use of a nanoindenter and QCM to study the high-speed tribology of microscopic contacts in fully characterized conditions. The indenter continuously tracks the contact radius, via the vertical contact stiffness, and measures the normal load and displacement. Transitions in the QCM frequency and bandwidth signals provide evidence of microslip for lubricated sapphire-ongold contacts. By modeling the dynamics and comparing to

${ }^{a)}$ Electronic mail: borovsky@stolaf.edu our data, we conclude that the reciprocating interface achieves full slip during half of each cycle, with a more fully stuck condition induced at higher loads.

We have previously reported on the integration of a QCM and nanoindenter. ${ }^{4}$ Figure 1 shows a diagram of the apparatus. The $5 \mathrm{MHz}$ quartz disks were mounted on a $x y z$ piezo scanner (NANOSCOPE® II, Digital Instruments) and driven with a commercial circuit (QCM 100, SRS). The oscillator circuit continuously measured the resonant frequency $( \pm 0.02 \mathrm{ppm})$ and conductance $( \pm 100 \mathrm{ppm})$ of the crystal. The conductance output (in volts) was converted to a full width at half maximum bandwidth by assuming a typical motional inductance of $10 \mathrm{mH}$. From the drive amplitude of $2.0 \mathrm{~V}_{\mathrm{pp}}$ and quality factors of 40000 to 80000 , we estimate oscillation amplitudes from 50 to $100 \mathrm{~nm}$ and peak surface velocities in the range of $2-4 \mathrm{~m} / \mathrm{s} .{ }^{9}$ We used a commercial indenter probe (TRIBOSCOPE®, Hysitron, Inc.) with a $800 \mu \mathrm{m}$ diameter spherical sapphire tip. This instrument is electrostatically actuated and uses force modulation (at $20 \mathrm{~Hz}$ in these experiments) to enable dynamic measurements of the contact stiffness normal to the substrate $k_{n} \cdot{ }^{10} \mathrm{We}$ calculate the contact radius $a_{c}$ from the relation $a_{c}=k_{n} / 2 E^{*}$, where $E^{*}$ is the reduced Young's modulus. ${ }^{11,12}$

The samples used were AT-cut quartz disks with electrodes consisting of a $10 \mathrm{~nm}$ thick chrome adhesion layer and a $100 \mathrm{~nm}$ gold outer layer (ICM, Inc.). An additional layer of gold $20 \mathrm{~nm}$ thick was deposited in vacuum at 5

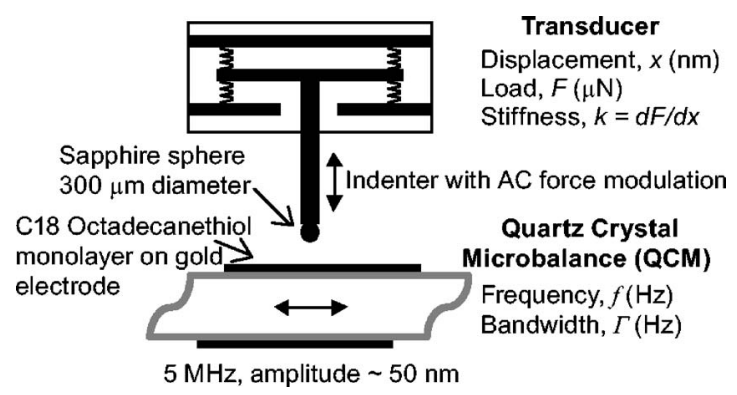

FIG. 1. Schematic diagram of the experimental setup. 

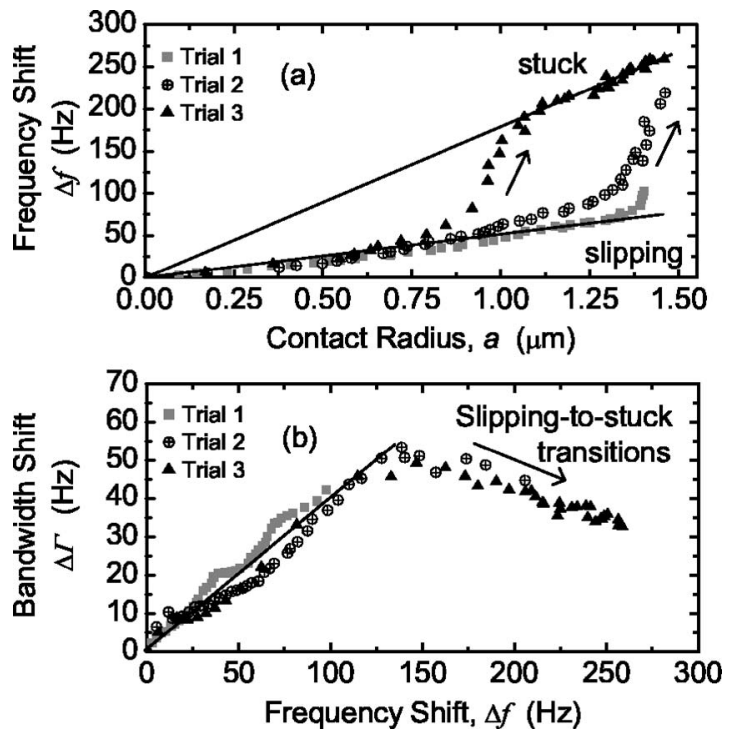

FIG. 2. (a) Plot of the QCM frequency shift vs contact radius for three consecutive tests at one location. We observe upward transitions in the frequency, interpreted as taking the interface from slipping to stuck conditions. (b) A plot of the QCM bandwidth shift vs frequency shift. During the transitions, the bandwidth of the resonance decreases, suggesting a decrease in sliding friction as more of the interface becomes stuck.

$\times 10^{-8}$ Torr. Octadecanethiol monolayers $\left[\mathrm{CH}_{3}\left(\mathrm{CH}_{2}\right)_{17} \mathrm{SH}\right.$, Sigma-Aldrich] were then deposited onto the electrodes by immersing the crystal in a $1 \mathrm{mM}$ absolute ethanol solution for $24 \mathrm{~h}$. Once prepared, the samples were tested at atmospheric pressure in a dry nitrogen environment with $1 \%$ relative humidity. During indentation tests, the tip was pressed into the sample with a staircase ramp cycle from $1 \mu \mathrm{N}$ to $5 \mathrm{mN}$ at an effective rate of $1 \mathrm{mN} / \mathrm{min}$. Average contact pressures in these experiments were near $1 \mathrm{GPa}$, with the load distributed among many individual contact points subject to plastic deformation and wear. ${ }^{5}$ Shifts in the QCM resonance parameters are proportional to the tangential forces at the interface, which are expected to scale with contact area. ${ }^{4,5}$ Therefore, contact radius is a more direct control parameter in these experiments than normal load.

Figure 2(a) shows the QCM frequency shift versus contact radius for three consecutive indentations at the same location on the sample. For clarity, only the loading portions of the tests are shown. We observe spontaneous transitions to much higher values of the frequency shift in two instances. We propose each transition marks a conversion at the interface from slipping to stuck conditions. The stuck contact is subject to higher elastic restoring forces, which increases the resonant frequency of the system. The data suggest two linear relationships, indicating the sliding and stuck cases, respectively. The frequency shift when sliding is about onethird of that when stuck.

Figure 2(b) shows the QCM bandwidth shift $\Delta \Gamma$ versus frequency shift $\Delta f$. We observe that during the transitions, the bandwidth decreases significantly as the frequency increases. This trend provides further evidence that slipping portions of the interface become stuck during the observed transitions. The loss of contact area subject to interfacial slip reduces friction and sharpens the resonance, while simultaneously increasing the contact stiffness and resonant frequency. We note that the bandwidth shift remains nonzero after the transition (compared to when the tip is out of contact), which suggests that some sliding friction remains
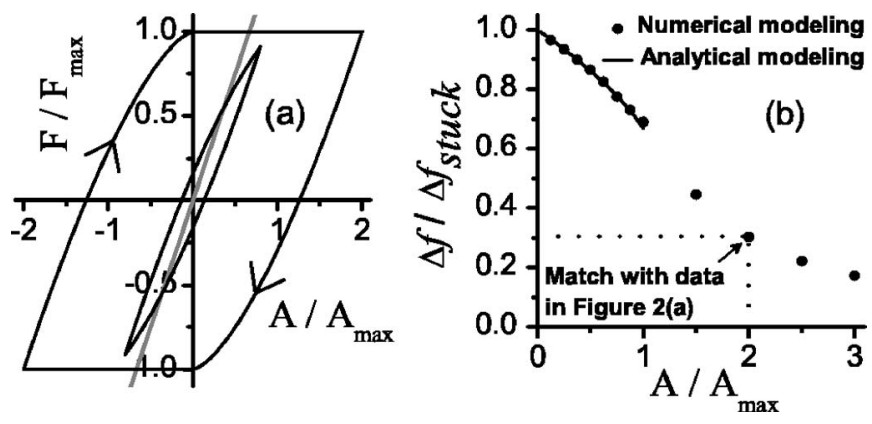

FIG. 3. (a) Theoretical hysteresis loops for microslip (tangential force vs displacement). At amplitudes greater than $A_{\max }$, the system experiences full slip with a frictional force $F_{\max }=\mu \mathrm{N}$. Loops are shown for $A=0.8 A_{\max }$ and $A=2.0 A_{\max }$. The gray line represents the fully stuck, totally elastic limit. (b) A normalized plot of QCM frequency shift vs displacement from a numerical simulation with microslip. The solid curve displays the results of an analytic solution for amplitudes less than $A_{\max }$, for which the microslip hysteresis loop can be modelled as an ellipse.

present or that other modes of dissipation, such as rolling friction, are significant aspects of the interaction.

The microslip theory of Mindlin et al. describes a reciprocating interface consisting of a central stuck portion surrounded by an outer annulus of slip. ${ }^{13,14}$ The slipping and stuck portions vary in size throughout the motion. During each half cycle, the slip annulus grows inward with tangential displacement from the endpoint of the motion. Microslip is generally expected to occur for sphere-on-flat geometries, due to the decrease in normal pressure at the outer regions of the contact. The total tangential force at the interface is the sum of the elastic force over the stuck portion and the classical (Coulombic) friction force over the slip annulus. This force is plotted in Fig. 3(a) as a function of normalized displacement, $x / A_{\max }$, for amplitudes $A=0.8 A_{\max }$ and $A$ $=2 A_{\max }$, where $A_{\max }$ is the point at which further displacement causes full slip at a constant frictional force $F_{\max }$ $=\mu \mathrm{N}$. The force-displacement curves trace hysteresis loops whose enclosed areas determine the frictional energy dissipated per cycle.

Overall, this family of hysteresis loops reveals the extent to which an interface subject to microslip is more compliant than its equivalent fully stuck interface. (For comparison, we show in Fig. 3(a) the linear force-displacement relationship for fully stuck conditions. In this case, the interface is totally elastic, dissipates no energy, and has a maximum stiffness.) Each hysteresis loop has a curved portion for leftward motion given by

$$
F=F_{\max }\left\{2\left(1+\frac{x-A}{2 A_{\max }}\right)^{3 / 2}-\left(1-\frac{A}{A_{\max }}\right)^{3 / 2}-1\right\} .
$$

The relationship is symmetric, so that $F^{\prime}(x)=-F(-x)$ describes the curved portion for rightward motion. Flat portions occur when full slip dictates $F=F_{\max }$. Denoting the entire hysteresis loop by $F_{\text {hys }}$, we may determine the shift in resonance parameters associated with this interaction by treating $F_{\text {hys }}$ as a perturbation to a mechanical model of the quartz crystal with effective parameters of mass $m$, spring stiffness $k$, and damping coefficient $b$,

$$
m \ddot{x}=-b \dot{x}-k x-F_{\text {hys }}(x) .
$$

Implementing a fourth-order Runge-Kutta numerical solution, we find that the frequency of oscillations for the undriven system follows the curve shown in Fig. 3(b). We have 
plotted the normalized frequency shift versus normalized amplitude. These results were found to be insensitive to the quality factor of the system for $Q=10000$ to 100000 . The size of the perturbation in the simulation was taken to be consistent with the experiment, disturbing the characteristic frequency to about $40 \mathrm{ppm}$.

The results of this numerical model may be compared to our observations of slipping-to-stuck transitions. Taking the ratio of the smaller to larger slope in Fig. 2(a), we find $\Delta f / \Delta f_{\text {stuck }}=0.30 \pm 0.03$. As shown in Fig. 3(b), this value is obtained at an amplitude of $(2.0 \pm 0.2) A_{\max }$, or twice what is needed to achieve full slip. Therefore, an oscillation amplitude well beyond the full-slip limit of microslip is necessary to account for the size of the observed transitions in resonant frequency. Referring to Fig. 3(a), we note that at this amplitude, the interface achieves full slip when the displacement crosses equilibrium and continues until the endpoint is reached. The interface spends half of each cycle in a state of full slip.

Within the microslip theory, $A_{\max }$ is given by $A_{\max }$ $=3 \mu \mathrm{N} / 16 G^{*} a_{c}$, where $G^{*}$ is the reduced shear modulus. ${ }^{14}$ For an order-of-magnitude estimate of $A_{\max }$, we may assume $\mu=0.1$ and insert typical values of $N=5 \mathrm{mN}, a_{c}=1.4 \mu \mathrm{m}$, and $G^{*}=14 \mathrm{GPa}$ to find $A_{\max } \sim 5 \mathrm{~nm}$. This result corresponds to a QCM oscillation amplitude of $2 A_{\max } \sim 10 \mathrm{~nm}$. This is a reasonable value for our quartz crystal at the drive level and quality factor reported above. ${ }^{9}$ Estimates for the mean shear stresses are $8 G^{*}\left(2 A_{\max }\right) / \pi a_{c}=250 \mathrm{MPa}$ while the contact is stuck, ${ }^{14}$ and $\mu \mathrm{N} / \pi a_{\mathrm{c}}^{2}=80 \mathrm{MPa}$ while slipping. This stress regime is routinely accessed by proximal probe techniques. ${ }^{15}$

When the oscillation amplitude is less than $A_{\max }$, any microslip hysteresis loop can be closely approximated by an ellipse parameterized by amplitude-dependent stiffness and damping coefficients: $\quad F_{\text {hys }} \sim k^{\prime}(A) x+b^{\prime}(A) \nu$, with $k^{\prime}$ $=F_{\text {hys }}(x=A) / A$ and $b^{\prime}=F_{\text {hys }}(x=0) / A w$, where $w$ is the unperturbed angular frequency at resonance. The mechanical model of Eq. (2) becomes a damped mass-and-spring system with the usual analytic solution. The resulting normalized frequency shift is shown as a solid line in Fig. 3(b) and agrees well with our numerical results. This agreement underscores the difficulty of distinguishing between mechanisms of friction. The response of the quartz resonance to an interaction involving classical microslip (velocityindependent friction) may be well described by a model incorporating a viscous damping coefficient (velocitydependent friction). This ambiguity is important to bear in mind in situations, as in Ref. 6, where an effective damping parameter is extracted from QCM data during an indentation test and is understood to reveal a velocity-dependent friction force at the interface. The most direct approach to distinguishing true velocity dependence from displacement hysteresis is to independently vary the amplitude and velocity of motion, but this is not possible with quartz crystals operated in resonance. Using an apparatus based on piezoelectric actuation off resonance may be more practical in this regard. ${ }^{8}$ However, such a scheme comes at the cost of the precise detection of lateral forces made possible by resonating the quartz crystal.

The results presented here show that the integration of a quartz microbalance with a probe capable of force, displacement, and contact stiffness measurements results in a powerful method of investigating interaction forces at high-speed microscopic contacts. The QCM frequency and bandwidth signals contain information about frictional energy loss as well as the state of relative motion at the interface. Interpretation of these signals is significantly improved by simultaneously tracking the contact radius with the indenter probe. Comparison of sliding and stuck conditions reveals that the sliding interface readily achieves full-slip conditions during its reciprocating motion, even at standard QCM drive levels and relatively high contact pressures. Therefore, experiments with this technique promise to elucidate fundamental aspects of sliding friction in the high-speed regime, such as the connection between frictional properties and molecular structure for molecularly thin lubricant films.

This work was supported by the National Science Foundation (Grant No. DMR-0523227), Research Corporation (Grant No. CC5608), and the Howard Hughes Medical Institute. We thank Hysitron, Inc. for customization of the TRIBOSCOPE $®$ software and for helpful consultation.

${ }^{1}$ The Handbook of Micro/Nanotribology, 2nd ed., edited by B. Bhushan (CRC, New York, 1999), pp. 5-7.

${ }^{2}$ R. Maboudian, W. R. Ashurst, and C. Carraro, Tribol. Lett. 12, 95 (2002).

${ }^{3}$ J. Krim, Am. J. Phys. 70, 890 (2002).

${ }^{4}$ A. Laschitsch and D. Johannsmann, J. Appl. Phys. 85, 3759 (1999).

${ }^{5}$ B. Borovsky, J. Krim, S. A. Syed Asif, and K. J. Wahl, J. Appl. Phys. 90, 6391 (2001).

${ }^{6}$ S. Berg and D. Johannsmann, Phys. Rev. Lett. 91, 145505 (2003).

${ }^{7}$ S. Berg, M. Ruths, and D. Johannsmann, Phys. Rev. E 65, 026119 (2002).

${ }^{8}$ E. Tocha, T. Stefanski, H. Schonherr, and G. J. Vancso, Rev. Sci. Instrum. 76, 083704 (2005).

${ }^{9}$ B. Borovsky, B. L. Mason, and J. Krim, J. Appl. Phys. 88, 4017 (2000).

${ }^{10}$ S. A. Syed Asif, K. J. Wahl, and R. J. Colton, Rev. Sci. Instrum. 70, 2408 (1999).

${ }^{11}$ K. L. Johnson, Contact Mechanics (Cambridge University Press, Cambridge, London, 1985), p. 92.

${ }^{12}$ In calculating the contact area, we used a reduced modulus of $E^{*}$ $=77 \mathrm{GPa}$ and a machine stiffness of $k_{m}=53.7 \mu \mathrm{N} / \mathrm{nm}$, consistent with the methods of Refs. 5 and 10. Our relatively low machine stiffness is due to the flexing of the structure suspending the quartz crystal.

${ }^{13}$ R. D. Mindlin, W. P. Mason, J. F. Osmer, and H. Deresiewicz, in Proceedings of the First U.S. National Congress of Applied Mechanics (ASME, New York, 1952), p. 203.

${ }^{14}$ K. L. Johnson, Contact Mechanics (Cambridge University Press, Cambridge, London, 1985), pp. 210-231.

${ }^{15}$ J. Krim, Sci. Am. 275(4), 74 (1996). 